\title{
Efectos de las técnicas de ejercicio terapéutico correctivo en la escoliosis idiopática del adolescente. Revisión sistemática
} Effects of corrective, therapeutic exercise techniques on
adolescent idiopathic scoliosis. A systematic review

Klgo. Luis Ceballos Laita ${ }^{a}$ Klga. Cristina Tejedor Cubillo ${ }^{b}$, Dra. Teresa Mingo Gómez ${ }^{a}$ y Dra. Sandra Jiménez del Barrio ${ }^{a}$

a. Escuela de

Fisioterapia, Campus Universitario Duques de Soria, Universidad de Valladolid, Soria, España.

b. Fisioterapeuta por cuenta ajena.

Correspondencia:

Klgo. Luis Ceballos Laita:

Luis.ceballos@uva.es

Financiamiento:

Ninguno.

Conflicto de intereses:

Ninguno que declarar.

Recibido: 10-11-2017

Aceptado: 19-2-2018

\section{RESUMEN}

El objetivo del estudio fue determinar los efectos de las técnicas de ejercicio terapéutico correctivo en sujetos con escoliosis idiopática del adolescente. Se realizó una revisión sistemática realizando búsquedas en Biblioteca Cochrane Plus, Pubmed, PEDro y SCOPUS. Se incluyeron estudios de pacientes con diagnóstico de escoliosis idiopática del adolescente, que consideraron, como variable independiente, ejercicio terapéutico correctivoy, como variables dependientes, síntomas, capacidad funcional, ángulo de Cobb y / u otros ángulos o asimetrías corporales. Se incluyeron un total de 9 ensayos clínicos controlados que utilizaron ejercicio terapéutico correctivo. El ejercicio terapéutico correctivo parece tener efectos positivos en la disminución de síntomas, mejora de la función y mejora de diferentes ángulos y asimetrías corporales. Sin embargo, son necesarios estudios con mejor calidad metodológica para confirmar estos resultados y determinar la mejor intervención mediante ejercicio terapéutico. Palabras clave: escoliosis, adolescente, kinesiología aplicada.

http: / / dx.doi.org/10.5546/ aap.2018.e582 Texto completo en inglés:

http: / / dx.doi.org/10.5546/ aap.2018.eng.e582

\section{INTRODUCCIÓN}

La deformidad espinal se denomina "escoliosis" cuando el raquis presenta una desviación frontal cuya magnitud de curva (ángulo de (obb) es mayor de $10^{\circ}$ y está asociada a la rotación de los cuerpos vertebrales. ${ }^{1}$ En concreto, la escoliosis idiopática del adolescente (EIA) es la deformidad espinal más común y es de gran importancia diagnóstica en la población pediátrica, especialmente, en la adolescencia. ${ }^{2}$

De forma general, se considera que entre el $2 \%$ y el $4 \%$ de los jóvenes de entre 10 y 16 años la presenta, lo que constituye el $84 \%-89 \%$ del total de sujetos afectados por escoliosis. ${ }^{3} \mathrm{Un}$ $10 \%$ de estas escoliosis progresan y se pueden asociar a otro tipo de anomalías orgánicas, sobre todo, neurológicas. ${ }^{4}$ Todas estas manifestaciones hacen que el diagnóstico y el tratamiento precoz sean fundamentales para la mejor calidad de vida.

Los signos característicos de la

Cómo citar: Ceballos Laita L, Tejedor Cubillo C, Mingo Gómez T, Jimenez del Barrio S. Efectos de las técnicas de ejercicio terapéutico correctivo en la escoliosis idiopática del adolescente. Revisión sistemática. Arch Argent Pediatr 2018;116(4):e582-e589.
EIA son las asimetrías, protuberancias y deformaciones torácicas. ${ }^{5}$ Pese a no ser un factor clínico principal, los pacientes con EIA pueden presentar dolor; son más proclives a desarrollarlo en mayores grados de ángulo de Cobb. ${ }^{6}$ Estudios de prevalencia han mostrado que el dolor se presenta entre el $27 \%$ y el $59 \%$ de los casos. ${ }^{5,7}$ Además, se pueden asociar otros problemas, como la restricción de la ventilación, la debilidad de la musculatura respiratoria, la disminución de la calidad de vida ${ }^{8}$ e, incluso, problemas psicológicos. ${ }^{9}$ 
Actualmente, según el consenso de EIA de $2016,{ }^{1}$ el seguimiento y el tratamiento de esta patología incluye la observación, el tratamiento con corsé o la cirugía. La Scoliosis Research Society (SRS) sugiere el tratamiento ortésico en las deformidades que superen los $25^{\circ}$ de ángulo de Cobb. El tratamiento quirúrgico se indica en sujetos con una curva con un ángulo de $\mathrm{Cobb}>45^{\circ}-50^{\circ}$. Respecto al tratamiento conservador, se han propuesto diversas intervenciones mediante ejercicio terapéutico en esta población y se ha recomendado su aplicación no solo en los casos en los que no esté indicada la cirugía, sino también como tratamiento combinado con otras medidas, como las ortésicas. El objetivo de estos ejercicios se basa en la disminución de los síntomas y la mejora de las capacidades funcionales y la calidad de vida. ${ }^{10-15}$ Si bien la principal medida en estos pacientes es la ortésica, no se conocen los efectos aislados del ejercicio terapéutico correctivo en pacientes con EIA; ${ }^{1}$ de hecho, la Scientific Society on Scoliosis Orthopaedic Rehabilitation and Treatment $(\mathrm{SOSORT})^{16}$ ha mostrado una escasez de evidencia en el tratamiento conservador de estos pacientes.

El objetivo de este estudio es determinar los efectos del ejercicio terapéutico correctivo en pacientes con diagnóstico de EIA mediante una revisión sistemática.

\section{MÉTODOS}

Se ha realizado una revisión sistemática siguiendo los criterios de la Declaración PRISMA (Preferred reporting items for systematic review and meta-analysis). ${ }^{17}$

\section{Criterios de elegibilidad}

Los criterios de elegibilidad de los artículos se establecieron según las recomendaciones descritas por SOSORT: ${ }^{18}$

- Ensayos clínicos controlados (ECC).

- Especificar que los sujetos de la muestra presentaran diagnóstico de EIA con un ángulo de Cobb de $10^{\circ}-45^{\circ}$.

- Muestra en edad pediátrica considerada en el país de realización del estudio. Según la Asociación Española de Pediatría, un 51,7\% de los países consideraban la edad pediátrica entre 0 y 18 años. ${ }^{19}$

- Tener como variable independiente una intervención basada en ejercicio terapéutico correctivo y compararla con un grupo placebo, control u otras técnicas no quirúrgicas.

- Tener como variables dependientes, al menos, una de las siguientes: síntomas, capacidad funcional, ángulo de Cobb y / u otros ángulos o asimetrías corporales.

Criterios de exclusión:

- Aplicación de cualquier intervención quirúrgica u ortésica.

\section{Búsqueda de artículos}

Se realizó la búsqueda de ECC en bases de datos reconocidas por la comunidad científica: Biblioteca Cochrane Plus, Pubmed (MEDLINE), Physiotherapy Evidence Database (PEDro) y SCOPUS. La búsqueda se realizó entre febrero y octubre de 2017. Se consideraron artículos escritos en inglés, francés o castellano y no se pusieron límites a la fecha de publicación.

Se utilizaron los términos descriptores médicos (Medical Subject Headings; MeSh, por sus siglas en inglés) scoliosis, physical therapy modalities, exercise y conservative treatment, en sus diferentes combinaciones dependiendo del buscador utilizado unidos por los operadores lógicos $O R$ y $A N D$. Además, se revisaron las listas de referencia de los artículos primarios con el objetivo de no perder estudios potencialmente relevantes.

\section{Identificación de los artículos}

Dos revisores independientes aplicaron los criterios de elegibilidad para seleccionar estudios potencialmente relevantes sobre la base del título y del resumen de los resultados encontrados tras la búsqueda en las diferentes bases de datos mencionadas anteriormente. Se estableció un consenso para la inclusión de los artículos. Tras esto, se obtuvieron los textos completos de los estudios que cumplieron los criterios de elegibilidad. Los datos fueron extraídos del mismo modo por dos revisores independientes. Se registró autor y año de la publicación, tamaño de la muestra, edad de los participantes, intervención, síntomas, ángulo de Cobb, ángulo cráneo-vertebral, rotación del tronco, asimetrías corporales, capacidad funcional, resistencia muscular, función pulmonar y resultados de cada estudio. Un tercer revisor resolvía las dudas o desacuerdos que podían surgir a la hora de seleccionar los estudios.

\section{Evaluación de la calidad metodológica}

Del mismo modo, la evaluación de la calidad metodológica de los ECC incluidos se realizó por dos personas independientes mediante la escala PEDro (Tabla 1), que evaluaba la calidad 
metodológica de los estudios, y se consideraron puntuaciones de $6-10$ como de alta calidad; de 4-5, calidad moderada, y de 0-3, de baja calidad, ${ }^{20}$ y mediante la clasificación de los niveles de evidencia de Oxford (Oxford Centre of EvidenceBased Medicine; OCEBM, por sus siglas en inglés) (Tabla 2), criterio de recomendación para la unificación de SOSORT. ${ }^{18}$

\section{RESULTADOS}

\section{Selección de los estudios}

Se obtuvieron un total de 1206 artículos entre las diferentes bases de datos analizadas $\mathrm{y}$, finalmente, se consideraron 15 para la revisión detallada. De los artículos revisados en detalle, uno ${ }^{21}$ fue excluido porque la muestra no presentaba diagnóstico de EIA, otros $\operatorname{dos}^{22,23}$ por utilizar órtesis en el grupo intervención, dos más ${ }^{24,25}$ por no basarse en ejercicio terapéutico, y un último artículo ${ }^{26}$ fue excluido por no tratarse de un ECC. Finalmente, se incluyeron un total de 9 ensayos clínicos para el estudio. El proceso de selección de los artículos se refleja en el diagrama de flujo (Figura 1).

\section{Características de los estudios}

Todos los artículos fueron ECC escritos en inglés. El rango de años de publicación fue desde 2012 el más antiguo a 2017 el más actual. El tamaño muestral de los estudios fue de 24 a

TABla 1. Escala PEDro

1. Los criterios de elección fueron especificados.

2. Los sujetos fueron asignados al azar a los grupos (en un estudio cruzado, los sujetos fueron distribuidos aleatoriamente a medida que recibían los tratamientos).

3. La asignación fue oculta.

4. Los grupos fueron similares al inicio en relación con los indicadores de pronóstico más importantes.

5. Todos los sujetos fueron cegados.

6. Todos los terapeutas que administraron la terapia fueron cegados.

7. Todos los evaluadores que midieron al menos un resultado clave fueron cegados.

8. Las medidas de al menos uno de los resultados clave fueron obtenidas de más del $85 \%$ de los sujetos inicialmente asignados a los grupos.

$\begin{array}{lll}\text { No } \square & \text { Sí } \square & \text { Dónde: } \\ \text { No } \square & \text { Sí } \square & \text { Dónde: } \\ \text { No } \square & \text { Sí } \square & \text { Dónde: } \\ \text { No } \square & \text { Sí } \square & \text { Dónde: } \\ \text { No } \square & \text { Sí } \square & \text { Dónde: } \\ \text { No } \square & \text { Sía } & \text { Dónde: } \\ \text { No } \square & \text { Sí } \square & \text { Dónde: } \\ \text { No } \square & \text { Sí } \square & \text { Dónde: } \\ & & \\ \text { No } \square & \text { Sí } \square & \text { Dónde: } \\ \text { No } \square & \text { Sí } \square & \text { Dónde: } \\ \text { No } \square & \text { Sí } \square & \text { Dónde: }\end{array}$

TABLA 2. Clasificación de los niveles de evidencia de Osford

\begin{tabular}{|c|c|c|}
\hline Grado de recomendación & Nivel de evidencia & Estudios de tratamiento \\
\hline \multirow[t]{3}{*}{$\mathrm{A}$} & $1 . \mathrm{a}$ & $\begin{array}{l}\text { RS con homogeneidad de EC controlados con asignación } \\
\text { aleatoria }\end{array}$ \\
\hline & 1.b & EC individual con intervalo de confianza estrecho \\
\hline & 1.c & $\begin{array}{l}\text { Eficiencia demostrada por la práctica clínica. Considera } \\
\text { cuando algunos pacientes mueren antes de ser evaluados }\end{array}$ \\
\hline \multirow[t]{5}{*}{$\mathrm{B}$} & 2.a & RS de estudios de dochortes, con homogeneidad \\
\hline & $2 . b$ & $\begin{array}{l}\text { Estudio de cohores individual con seguimiento inferior } \\
\text { a } 80 \% \text { (incluye EC de baja calidad) }\end{array}$ \\
\hline & 2.c & Estudios ecológicos o de resultados de salud \\
\hline & 3.a & RS de estudios de casos y controles con homogeneidad \\
\hline & 3.b & Estudios de casos y controles individuales \\
\hline $\mathrm{C}$ & 4 & $\begin{array}{l}\text { Series de casos, estudios de cohortes y de casos y } \\
\text { controles de baja calidad }\end{array}$ \\
\hline $\mathrm{D}$ & 5 & $\begin{array}{l}\text { Opinión de expertos sin evaluación crítica explícita, } \\
\text { ni basada en fisiología, ni en trabajo de investigación } \\
\text { juicioso, ni en "principios fundamentales" }\end{array}$ \\
\hline
\end{tabular}


110 participantes. La suma de las muestras de los ECC incluidos mostró un total de 459 pacientes, divididos en 2 grupos, a excepción de un estudio que presentó $3 .{ }^{27}$ Todos los sujetos incluidos en los grupos fueron sujetos de edad pediátrica y con diagnóstico de EIA.
Todos los estudios incluidos presentaron como variable independiente el ejercicio terapéutico correctivo, 4 de ellos bajo el término de ejercicios de Schroth, ${ }^{27-30}$ y los 5 restantes bajo diferentes términos que comprendían ejercicio terapéutico basado en la autocorrección y la estabilización

Figura 1. Diagrama de flujo: Proceso de selección de los artículos

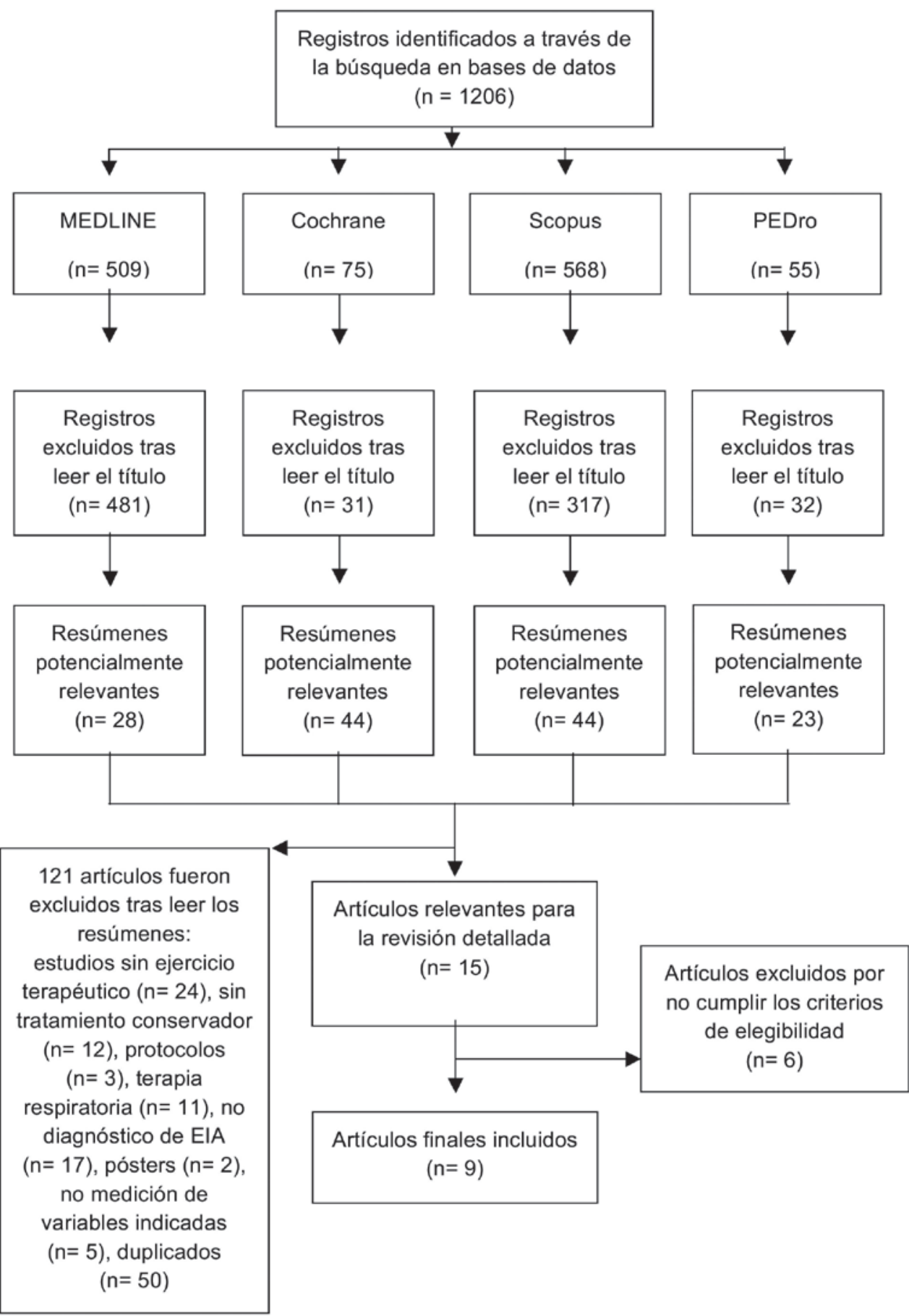

EIA: escoliosis idiopática del adolescente. 
vertebral. ${ }^{31-35}$ El período de intervención fue muy variable: 3 estudios realizaron una intervención de entre 8 y 12 semanas, ${ }^{30,32,35} 4$ realizaron intervenciones de 6 meses $^{27-29-33}$ y 2, de un año de duración. ${ }^{31,34}$

Con respecto a las variables dependientes consideradas, 6 estudios evaluaron los síntomas a través de los cuestionarios Scoliosis Research Society 22 (SRS-22), ${ }^{28,34}$ Scoliosis Research Society 23 (SRS-23), ${ }^{27}$ Numeric Pain Rating Scale (NPRS) ${ }^{32,33} \mathrm{y}$ del Functional Rating Index (FRI). ${ }^{35}$

Seis estudios evaluaron parámetros posturales, como el ángulo de Cobb a través de radiografías anteroposteriores del tronco en posición de bipedestación, ${ }^{27,29-31,34}$ el ángulo craneovertebral medido mediante fotogrametría sagital en posición sedente, ${ }^{35}$ el ángulo de rotación de tronco registrado mediante inclinometría ${ }^{27,34}$ y/o asimetrías corporales mediante la utilización de proyección estereográfica ${ }^{35} \mathrm{o}$ de una regla rígida. ${ }^{27}$

Seis estudios evaluaron la capacidad funcional de los sujetos incluidos mediante los cuestionarios SRS-22, 28,34 SRS-23, ${ }^{27}$ Oswestry Low Back Pain Disability Questionnaire (OSW), Patient-Specific Functional Scale (PSFS) ${ }^{32,33}$ y del FRI. ${ }^{35}$

En cuanto a la variable resistencia muscular, 2 estudios evaluaron la resistencia de la musculatura extensora de la espalda a través del Prone-double-Leg-Raise Test (PDLRT) ${ }^{32}$ y del Biering-Sorensen test (BME -back muscle endurance-). ${ }^{28}$

Dos estudios evaluaron la percepción global tras la intervención mediante el Global Rating of Change (GROC). ${ }^{32,33}$

Un solo articulo evaluó la función pulmonar mediante la realización de test de función pulmonar que medían Vital Capacity (VC), PeakFlow (PEF), Force expiratory Volume 1 (FEV1), Forced Vital Capacity (FVC) y la relación FEV1/ FVC. ${ }^{31}$
Calidad metodológica de los estudios incluidos

Ocho de los estudios obtuvieron puntuaciones iguales a 6 o superiores en la escala PEDro, lo cual significó un nivel de calidad alto. Dos estudios presentaron una puntuación igual a 5, lo cual significó un nivel de calidad moderado. Se presentaron aspectos mejorables, tales como la falta de cegamiento de pacientes y evaluadores o la presentación de los resultados de todos los sujetos del estudio. La puntuación detallada de la escala PEDro se muestra en la Tabla 3.

Todos los estudios presentaron un nivel de evidencia $1 b,{ }^{36}$ lo cual se correspondió con un nivel de recomendación aconsejable, evidencia moderada de que la medida era efectiva y los beneficios eran superiores a los perjuicios, proporcionado por ensayos clínicos aleatorizados con muy bajo riesgo de sesgos.

\section{Eficacia terapéutica}

Los resultados obtenidos tras cada uno de los tratamientos aplicados en los ensayos incluidos en esta revisión se muestran detalladamente respecto a cada una de las variables dependientes en la Tabla 4.

\section{DISCUSIÓN}

El objetivo de esta revisión fue determinar los efectos del ejercicio terapéutico correctivo en pacientes con diagnóstico de EIA.

Los resultados de los artículos incluidos mostraron que el ejercicio terapéutico había sido efectivo en la disminución de síntomas, ángulo de Cobb, ángulo craneovertebral, rotación del tronco y asimetrías corporales, también en la mejora de la resistencia muscular, la función pulmonar y la capacidad funcional de los pacientes con EIA. ${ }^{27-35}$

Con frecuencia, los síntomas en la EIA se asocian a procesos tumorales, inflamatorios o disfunciones viscerales; esto excluiría el

Tabla 3. Calidad metodológica. Puntuación en la escala PEDro

\begin{tabular}{|c|c|c|c|c|c|c|c|c|c|c|c|}
\hline Artículo & 1 & 2 & 3 & 4 & 5 & 6 & 7 & 8 & 9 & 10 & TOTAL \\
\hline Diab et al., 2012 & SÍ & SÍ & SÍ & $\mathrm{NO}$ & $\mathrm{NO}$ & $\mathrm{NO}$ & SÍ & SÍ & SÍ & SÍ & $7 / 10$ \\
\hline Monticone et al., 2014 & Sí & SÍ & Sí & SÍ & $\mathrm{NO}$ & SÍ & SÍ & $\mathrm{NO}$ & Sí & SÍ & $8 / 10$ \\
\hline Zapata et al., 2015 & Sí & $\mathrm{NO}$ & SÍ & $\mathrm{NO}$ & $\mathrm{NO}$ & SÍ & $\mathrm{NO}$ & $\mathrm{NO}$ & Sí & SÍ & $5 / 10$ \\
\hline Schreiber et al., 2015 & SÍ & SÍ & SÍ & $\mathrm{NO}$ & $\mathrm{NO}$ & SÍ & Sí & SÍ & SÍ & SÍ & $8 / 10$ \\
\hline Kuru et al., 2016 & SÍ & SÍ & SÍ & $\mathrm{NO}$ & $\mathrm{NO}$ & NO & SÍ & NO & Sí & SÍ & $6 / 10$ \\
\hline Schreiber et al., 2016 & SÍ & Sí & SÍ & $\mathrm{NO}$ & $\mathrm{NO}$ & SÍ & SÍ & SÍ & Sí & Sí & $8 / 10$ \\
\hline Kim et al., 2016 & Sí & NO & Sí & NO & NO & NO & SÍ & Sí & Sí & Sí & $6 / 10$ \\
\hline Zapata et al., 2017 & Sí & NO & Sí & $\mathrm{NO}$ & NO & Sí & NO & $\mathrm{NO}$ & Sí & Sí & $5 / 10$ \\
\hline Kumar et al., 2017 & SÍ & SÍ & SÍ & NO & $\mathrm{NO}$ & SÍ & SÍ & SÍ & SÍ & SÍ & $8 / 10$ \\
\hline
\end{tabular}


tratamiento únicamente conservador. Los estudios incluidos en esta revisión no presentaban este tipo de pacientes y, tras la intervención, mostraron una disminución en los síntomas. La bibliografía no es unánime en la génesis de síntomas en la EIA. ${ }^{6}$ Algunos autores lo han atribuido a la carga asimétrica que provoca una degeneración prematura de facetas y discos intervertebrales y acortamiento de musculatura. ${ }^{6,37,38}$ Sin embargo, no se conoce con exactitud el mecanismo por el que se habría mejorado esta variable, que puede venir determinado por la mejora de la flexibilidad de la columna y de los tejidos adyacentes ${ }^{39}$ o por la propia liberación de sustancias moduladoras del dolor por el propio ejercicio. ${ }^{40}$

Un artículo observó mejoras en la capacidad pulmonar. ${ }^{31}$ Las alteraciones respiratorias son más frecuentes conforme aumenta el ángulo de Cobb, ${ }^{41}$ por lo que, en estos pacientes, estarían indicadas las órtesis o incluso la cirugía. ${ }^{1}$ Este dato debe considerarse con precaución, ya que solo se ha observado en un estudio.

TABLA 4. Sintesis de resultados

\begin{tabular}{|c|c|c|c|c|c|}
\hline Autor & $\mathbf{N}$ & $\begin{array}{l}\text { Edad (años) } \\
\text { Media } \pm \text { DE }\end{array}$ & Intervención & Variables & Resultados \\
\hline Diab et al., 2012 & G1: 38 & $14,5 \pm 1,3$ & $\begin{array}{c}\text { G2: grupo autocorrección } \\
\text { postural }\end{array}$ & $\begin{array}{l}\text { Ángulo craneovertebral, inclinación } \\
\text { de tronco, lordosis, cifosis, } \\
\text { desequilibrio, desviación lateral, } \\
\text { torsión pélvica y rotación FRI }\end{array}$ & $\begin{array}{l}\text { G1-G2: } p<0,05 ; \text { mejora G2 tras } \\
10 \text { semanas y } 3 \text { meses } \\
\text { G1-G2: } p<0,05 \text {; mejora G2 a } \\
3 \text { meses }\end{array}$ \\
\hline \multirow[t]{2}{*}{ Monticone et al., 2014} & G1: 55 & $12,5 \pm 1,1$ & G1: grupo control & Ángulo de Cobb, ATR, SRS-22 & $\begin{array}{l}\text { G1-G2: } \mathrm{p}<0,001 ; \text { mejora } \mathrm{G} 2 \\
\text { tras tto. y a } 12 \text { meses }\end{array}$ \\
\hline & G2: 55 & $12,4 \pm 1,1$ & G2: grupo estabilización & & \\
\hline \multirow[t]{2}{*}{ Zapata et al., 2015} & G1: 17 & $15,7 \pm 2,0$ & G1: grupo estabilización & NPRS, PSFS & $\begin{array}{l}\text { G1-G2: } p<0,05 ; \text { mejora } \mathrm{G} 1 \text { tras } \\
8 \text { semanas }\end{array}$ \\
\hline & G2: 17 & $14,1 \pm 2,0$ & $\begin{array}{l}\text { G2: grupo estabilización } \\
\text { no supervisado }\end{array}$ & OSW, PDLRT & $\begin{array}{l}G 1: p=0,001 / G 2: p=0,001 \\
G 1: p=0,001 / G 2: p=0,001\end{array}$ \\
\hline \multirow[t]{2}{*}{ Schreiber et al.,2015 } & G1: 25 & $12,7 \pm 1,5$ & G1: grupo control & BME test & $\begin{array}{l}\text { G1-G2: } p=0,04 ; \text { mejora } G 2 \text { tras } \\
3 \text { meses }\end{array}$ \\
\hline & G2: 25 & $12,7 \pm 1,2$ & G2: grupo Schroth & SRS-22 & $\begin{array}{l}\text { G1-G2: } \mathrm{p}<0,05 ; \text { mejora G2 } \\
\text { tras } 3 \text { meses los síntomas y la } \\
\text { función }\end{array}$ \\
\hline \multirow[t]{3}{*}{ Kuru et al., 2016} & G1: 15 & $12,9 \pm 1,4$ & G1: grupo Schroth & Ángulo de Cobb & $\begin{array}{l}\text { G1-G2-G3: } p=0,003 \text {; mejora } G 1 \\
\text { a } 24 \text { semanas }\end{array}$ \\
\hline & G2: 15 & $13,1 \pm 1,7$ & $\begin{array}{l}\text { G2: grupo Schroth no } \\
\text { supervisado }\end{array}$ & $\begin{array}{l}\text { Ángulo de rotación, altura de la } \\
\text { giba, asimetría de la cintura }\end{array}$ & $\begin{array}{l}\text { G1-G2-G3: } \mathrm{p}<0,01 ; \text { mejora G1 } \\
\text { tras } 6 \text { y } 24 \text { semanas }\end{array}$ \\
\hline & G3: 15 & $12,8 \pm 1,2$ & G3: control & & \\
\hline \multirow[t]{2}{*}{ Schreiber et al., 2016} & G1: 25 & $13,5(12,7-14,2)$ & G1: grupo Schroth & Curva mayor & $\begin{array}{l}\text { G1-G2: } p=0,006 \text {; mejora } \mathrm{G} 1 \\
\text { tras } 6 \text { meses }\end{array}$ \\
\hline & G2: 25 & $13,3(12,7-13,9)$ & G2: grupo control & Suma de curvas & $\begin{array}{l}\text { G1-G2: } \mathrm{p}=0,048 ; \text { mejora G1 } \\
\text { tras } 6 \text { meses }\end{array}$ \\
\hline \multirow[t]{2}{*}{ Kim et al., 2016} & G1: 12 & $15,3 \pm 0,8$ & G1: grupo Schroth & $\begin{array}{l}\text { Ángulo de Cobb, distribución de } \\
\text { peso al lado convexo y cóncavo }\end{array}$ & $\begin{array}{l}\text { G1-G2: } p<0,05 ; \text { mejora } G 1 \text { tras } \\
12 \text { semanas }\end{array}$ \\
\hline & G2: 12 & $15,6 \pm 1,1$ & G2: grupo Pilates & & \\
\hline \multirow[t]{2}{*}{ Zapata et al., 2017} & G1: 17 & $15,5 \pm 2,2$ & G1: grupo estabilización & NPRS y GROC & $\begin{array}{l}\text { G1-G2: } p<0,05 ; \text { mejora } \mathrm{G} 1 \text { tras } \\
6 \text { meses }\end{array}$ \\
\hline & G2: 17 & $14,0 \pm 2,0$ & $\begin{array}{l}\text { G2: grupo estabilización } \\
\text { no controlado }\end{array}$ & OSW y PSFS & G1: $p<0,001 / G 2: p<0,001$ \\
\hline \multirow[t]{2}{*}{ Kumar et al., 2017} & G1: 18 & $12,1 \pm 1,7$ & G1: grupo control & Ángulo de Cobb, FVC, FEV1, VC & $\begin{array}{l}\text { G1-G2: } p<0,001 ; \text { mejora G2 } \\
\text { tras un año }\end{array}$ \\
\hline & G2: 18 & $11,5 \pm 1,4$ & $\begin{array}{l}\text { G2: grupo autocorrección } \\
\text { postural }\end{array}$ & PEF & $\mathrm{G} 1: \mathrm{p}<0,05 / \mathrm{G} 2: \mathrm{p}<0,05$ \\
\hline
\end{tabular}

G: grupo; p: valor estadístico; ATR: ángulo de rotación de tronco; BME: resistencia de la musculatura de la espalda;

FEV1: volumen espiratorio forzado; FRI: índice de clasificación funcional; FVC: capacidad vital forzada;

GROC: escala global de clasificación del cambio; NPRS: escala numérica de clasificación del dolor;

OSW: cuestionario Oswestry de dolor de espalda revisado; PDLRT: test de elevación de las piernas en prono;

PEF: flujo espiratorio máximo; PSFS: escala funcional específica del paciente;

SRS-22r: cuestionario 22 de la Sociedad de Investigación de la Escoliosis para el Paciente;

SRS-23: cuestionario 23 de la Sociedad de Investigación de la Escoliosis para el Paciente; VC: capacidad vital. 
Otro de los hallazgos observados en los artículos incluidos es la mejora en las variables posturales. El hecho de haber incidido en la autocorrección y el control postural habría ayudado a conseguir la elongación de los tejidos de las áreas cóncavas y la modificación de la tensión de los tejidos de las áreas convexas. Además, un aspecto clave en el entendimiento biomecánico de estos ejercicios es la activación de la musculatura estabilizadora de la columna, como los multífidos, los rotadores cortos y el transverso abdominal, entre otros, lo cual ayudaría a la mejora del equilibrio postural y la función muscular, y pueden atribuirse también mejoras en los test de resistencia muscular. ${ }^{42,43}$

Los resultados en conjunto muestran una mejora postural que radica en la capacidad de mantener la autocorrección y el control postural a lo largo del tiempo. Estudios previos avalan los beneficios del ejercicio terapéutico al demostrar que los ejercicios correctivos pueden evitar el empeoramiento de las curvas ${ }^{14}$ y pueden llegar a evitar el uso de la órtesis ${ }^{11}$ y de la cirugía. ${ }^{44}$

Las mejoras en la capacidad funcional y la calidad de vida observadas tras la aplicación de ejercicio terapéutico en pacientes con EIA podrían atribuirse a una disminución en los síntomas que, junto con la mejora en los parámetros posturales, podrían haber influido en la autoimagen de los pacientes y en su satisfacción personal.

Con respecto al número de sesiones por semana, aspecto controvertido en otros trabajos de investigación, ${ }^{45}$ no se ha encontrado una uniformidad en el número de sesiones ni en su duración, además de considerar que, en algunos estudios, se realizaban sesiones presenciales y no presenciales. Cabe destacar que la capacidad de aprendizaje y de control difiere de un sujeto a otro, por lo tanto, es difícil hacer una estimación acerca de la intensidad y duración de la intervención.

Este estudio no evalúa los efectos combinados de las medidas ortésicas con el ejercicio terapéutico; se considera que, clínicamente, no se utiliza una única técnica de tratamiento aislada y es este último una medida coadyuvante para el abordaje de la EIA.

\section{LIMITACIONES}

Una de las limitaciones principales fue el escaso número de artículos incluidos, lo que indicó una reducida evidencia acerca del ejercicio terapéutico en el manejo de esta patología.

Además, la metodología de algunos artículos no se detallaba de forma concreta, lo que dificultó la evaluación de la calidad metodológica. Todos ellos presentaron aspectos metodológicos mejorables, y se destacó la falta de cegamiento de pacientes y evaluadores o la presentación de los resultados de todos los sujetos del estudio.

Como se recomienda en el consenso de 2014 entre SOSORT y SRS, ${ }^{18}$ las diversas herramientas de medición de las variables dependientes hacen dificultosa la comparación entre los estudios.

Además, la baja calidad metodológica de algunos estudios no ha permitido la inclusión de algunas técnicas de ejercicio, como la reeducación postural global y las técnicas de Klapp, que clínicamente se emplean en estos pacientes.

\section{CONCLUSIÓN}

Los resultados de esta revisión parecen indicar los efectos positivos del tratamiento de la EIA con ejercicio terapéutico basado en el método Schroth o en ejercicios de estabilización. Muestran una disminución de los síntomas, mejora de la función y mejora de los ángulos vertebrales y asimetrías del tronco. No es posible describir el mejor tiempo de intervención ni el número de sesiones por semana, así como el tiempo de dedicación de cada sesión. Por lo tanto, se ve necesaria la realización de estudios de mejor calidad metodológica acerca del ejercicio terapéutico que midan variables tanto clínicas como de imagen para poder obtener resultados concluyentes.

\section{REFERENCIAS}

1. Gacitúa MV, González MC, Sanz C, et al. Consenso de escoliosis idiopática del adolescente. Arch Argent Pediatr 2016; 114(6):585-94.

2. Chin KR, Price JS, Zimbler S. A guide to early detection of scoliosis. Contemp Pediatr 2001; 18(9):77-103.

3. Schlösser TP, van der Heijden GJ, Versteeg AL, et al. How "idiopathic" is adolescent idiopathic scoliosis? A systematic review on associated abnormalities. PLoS One 2014; 9(5):e97561.

4. Reamy BV,Slakey J. Adolescent Idiopatic Scoliosis: Review and Current Concepts. Am Fam Physician 2001; 64(1):111-6.

5. Sato T, Hirano T, Ito T, et al. Back pain in adolescents with idiopathic scoliosis: epidemiological study for 43,630 pupils in Niigata City, Japan. Eur Spine J 2011; 20(2):274-9.

6. Gotfryd AO, Franzin FJ, Poletto PR, et al. Pain assessment in patients with adolescent idiopathic scoliosis at different stages of disease evolution. MedicalExpress (São Paulo. Online) 2014; 1(4):170-3.

7. Theroux J, Le May S, Fortin C, et al. Prevalence and management of back pain in adolescent idiopathic scoliosis patients: A retrospective study. Pain Res Manag 2015; 20(3):153-7.

8. Martínez-Llorens J, Ramírez M, Colomina MJ, et al. Muscle dysfunction and exercise limitation in adolescent idiopathic scoliosis. Eur Respir J 2010; 36(2):393-400.

9. Trobisch P, Suess O, Schwab F. Idiopathic scoliosis. Dtsch Arztebl Int 2010; 107(49):875-83. 
10. Berdishevsky H, Lebel VA, Bettany-Saltikov J, et al. Physiotherapy scoliosis-specific exercises-a comprehensive review of seven major schools. Scoliosis Spinal Disord 2016; 11:20.

11. Negrini S, Zaina F, Romano M, et al. Specific exercises reduce brace prescription in adolescent idiopathic scoliosis: A prospective controlled cohort study with worst-case analysis. J Rehabil Med 2008; 40(6):451-5.

12. Fusco C, Zaina F, Atanasio S, et al. Physical exercises in the treatment of adolescent idiopathic scoliosis: an updated systematic review. Physiother Theory Pract 2011; 27(1):80114.

13. YangJM, LeeJH, LeeDH. Effects of consecutive application of stretching, Schroth, and strengthening exercises on Cobb's angle and the rib hump in an adult with idiopathic scoliosis. J Phys Ther Sci 2015; 27(8):2667-9.

14. Negrini S, Fusco C, Minozzi S, et al. Exercises reduce the progression rate of adolescent idiopathic scoliosis: results of a comprehensive systematic review of the literature. Disabil Rehabil 2008; 30(10):772-85.

15. Weiss HR, Moramarco MM, Borysov M, et al. Postural rehabilitation for adolescent idiopathic scoliosis during growth. Asian Spine J 2016; 10(3):570-81.

16. Negrini S, Aulisa AG, Aulisa L, et al. 2011 SOSORT guidelines: Orthopaedic and Rehabilitation treatment of idiopathic scoliosis during growth. Scoliosis 2012; 7(1):3.

17. Moher D, Shamseer L, Clarke M, et al. Preferred reporting items for systematic review and meta-analysis protocols (PRISMA-P) 2015 statement. Syst Rev 2015; 4(1):1.

18. Negrini S, Hresko TM, O'Brien JP, et al. Recommendations for research studies on treatment of idiopathic scoliosis: Consensus 2014 between SOSORT and SRS non-operative management committee. Scoliosis 2015; 10(1):8.

19. Asociación Española de Pediatría de Atención Primaria. Pediatría en Europa: organización y formación. [Acceso: 20 de febrero de 2018]. Disponible en: https: / / www.aepap. org/grupos / grupo-de-relaciones-con-pediatria-europea / pediatria-en-europa.

20. Ajimsha MS, Al-Mudahka NR, Al-Madzhar JA. Effectiveness of myofascial release: Systematic review of randomized controlled trials. J Bodyw Mov Ther 2015; 19(1):102-12.

21. Dantas DS, De Assis S, Baroni M, et al. Klapp method effect on idiopathic scoliosis in adolescents : blind randomized controlled clinical trial. J Phys Ther Sci 2017; 29(1):1-7.

22. Gür G, Ayhan C, Yakut Y. The effectiveness of core stabilization exercise in adolescent idiopathic scoliosis: A randomized controlled trial. Prosthet Orthot Int 2017; 41(3):303-10.

23. Plewka B, Sibinski M, Synder M, etal. Clinical assessment of the efficacy of SpineCor brace in the correction of postural deformities in the course of idiopathic scoliosis. Pol Orthop Traumatol 2013; 78:85-9.

24. Weiss H-R, Hollaender M, Klein R. ADL based scoliosis rehabilitation--the key to an improvement of timeefficiency? Stud Health Technol Inform 2006; 123:594-8.

25. Lapuente JP, Sastre S, Barrios C. Idiopathic scoliosis under 30 degrees in growing patients. A comparative study of the F.E.D. method and other conservative treatments. Stud Health Technol Inform 2002; 88:258-69.

26. Rigo M, Quera-Salvá G, Villagrasa $M$, et al. Scoliosis intensive out-patient rehabilitation based on Schroth method. Stud Health Technol Inform 2008; 135:208-27.

27. Kuru T, Yeldan I, Dereli EE, et al. The efficacy of threedimensional Schroth exercises in adolescent idiopathic scoliosis: a randomised controlled clinical trial. Clin Rehabil 2016; 30(2):181-90.

28. Schreiber S, Parent EC, Moez EK, et al. The effect of Schroth exercises added to the standard of care on the quality of life and muscle endurance in adolescents with idiopathic scoliosis—an assessor and statistician blinded randomized controlled trial: "SOSORT 2015 Award Winner." Scoliosis 2015; 10(1):24.

29. Schreiber S, Parent EC, Moez EK, et al. Schroth Physiotherapeutic Scoliosis-Specific Exercises Added to the Standard of Care Lead to Better Cobb Angle Outcomes in Adolescents with Idiopathic Scoliosis - an Assessor and Statistician Blinded Randomized Controlled Trial. PLoS One 2016; 11(12): $\mathrm{e} 0168746$.

30. Kim G, HwangBo P. Effects of Schroth and Pilates exercises on the Cobb angle and weight distribution of patients with scoliosis. J Phys Ther Sci 2016; 28(3):1012-5.

31. Kumar A, Kumar S, Sharma V, et al. Efficacy of Task Oriented Exercise Program Based on Ergonomics on Cobb's Angle and Pulmonary Function Improvement in Adolescent Idiopathic Scoliosis- A Randomized Control Trial. J Clin Diagnostic Res 2017; 11(8):1-4.

32. Zapata KA, Wang-Price SS, Sucato DJ, et al. Spinal Stabilization Exercise Effectiveness for Low Back Pain in Adolescent Idiopathic Scoliosis. Pediatr Phys Ther 2015; 27(4):396-402.

33. Zapata KA, Wang-Price SS, Sucato DJ. Six-Month Followup of Supervised Spinal Stabilization Exercises for Low Back Pain in Adolescent Idiopathic Scoliosis. Pediatr Phys Ther 2017; 29(1):62-6.

34. Monticone M, Ambrosini E, Cazzaniga D, et al. Active self-correction and task-oriented exercises reduce spinal deformity and improve quality of life in subjects with mild adolescent idiopathic scoliosis. Results of a randomised controlled trial. Eur Spine J 2014; 23(6):1204-14.

35. Diab AA. The role of forward head correction in management of adolescent idiopathic scoliotic patients: a randomized controlled trial. Clin Rehabil 2012; 26(12):112332.

36. Marzo-Castillejo M, Viana-Zulaica C. Calidad de la evidencia y grado de recomendación. Guias Clínicas 2007; 7(Supl 1):6.

37. Danielsson AJ, Romberg K, Nachemson AL. Spinal Range of Motion, Muscle Endurance, and Back Pain and Function at Least 20 Years After Fusion or Brace Treatment for Adolescent Idiopathic Scoliosis: a case-control study. Spine (Phila Pa 1976) 2006; 31(3):275-83.

38. Bunnell WP. The natural history of idiopathic scoliosis before skeletal maturity. Spine (Phila Pa 1976) 1986;11(8):7736.

39. Smorgick Y, Mirovsky Y, Baker KC, et al. Predictors of back pain in adolescent idiopathic scoliosis surgical candidates. J Pediatr Orthop 2013; 33(3):289-92.

40. Louw A, Diener I, Butler DS, et al. The effect of neuroscience education on pain, disability, anxiety, and stress in chronic musculoskeletal pain. Arch Phys Med Rehabil 2011; 92(12):2041-56

41. Dos Santos Alves VL, Stirbulov R, Avanzi O. Impact of a physical rehabilitation program on the respiratory function of adolescents with idiopathic scoliosis. Chest 2006; 130(2):500-5.

42. Koumantakis GA, Watson PJ, Oldham JA. Trunk muscle stabilization training plus general exercise versus general exercise only: randomized controlled trial of patients with recurrent low back pain. Phys Ther 2005; 85(3):209-25.

43. Koumantakis GA, Watson PJ, Oldham JA. Supplementation of general endurance exercise with stabilisation training versus general exercise only. Physiological and functional outcomes of a randomised controlled trial of patients with recurrent low back pain. Clin Biomech (Bristol, Avon) 2005; 20(5):474-82.

44. Hawes MC. The use of exercises in the treatment of scoliosis: An evidence-based critical review of the literature. Pediatr Rehabil 2003; 6(3-4):171-82.

45. Negrini A, Verzini N, ParziniS, etal. Role of physical exercise in the tratment of mild idiopathic adolescent scoliosis. Eur Med Phis 2001; 37(3):181-90. 